Cuadernos de Gobierno y Administración Pública ISSN: e-2341-4839

http://dx.doi.org/10.5209/CGAP.64616

\title{
Reforma del sector de la seguridad en el Líbano: Consociacionalismo, securitización o democratización
}

\author{
Francisco Javier Lion Bustillo ${ }^{1}$
}

Recibido: 18/02/2019/ Aceptado: 07/052019

Resumen. Tradicionalmente, las Fuerzas Armadas Libanesas (FAL) han servido más para el control de la población que para la defensa del país. Por su parte, tanto las FAL como las numerosas agencias de seguridad existentes han sido distribuidas entre los distintos partidos de acuerdo con cuotas de afiliación confesional, aplicando el modelo consociacional característico del Líbano. Además, existen distintos actores no estatales dotados de un importante armamento, si bien algunos de ellos (como es el caso de Hezbollah) colaboran con las FAL componiendo un modelo de gobernanza bastante peculiar. Desde la salida de las tropas sirias en 2005, el país se ha visto dividido en lo relativo a cómo afrontar una reforma del sector de la seguridad. Mientras algunos actores han querido mantener el statu quo (con el apoyo sirio o iraní), otros deseaban únicamente el desarme de Hezbollah (respaldados por Estados Unidos y Arabia Saudí). El objetivo de este trabajo consiste en evaluar hasta qué punto las reformas efectuadas han contribuido a la creación de un sector de la seguridad ajustado a las exigencias de un régimen democrático, capaz de garantizar la paz interior, la neutralidad política y la defensa del país. Palabras clave: reforma del sector de la seguridad, Líbano, consociacionalismo, Fuerzas Armadas libanesas, fronteras.

\section{[en] Security Sector Reform in Lebanon: Consotiationalism, securitization or democratization}

\begin{abstract}
Traditionally, the Lebanese Armed Forces (LAF) have been more devoted to the control of Lebanese population than to national defence. At the same time, the control of both the LAF and the many other existing security agencies have been divided among the different political parties according to quotas of confessional affiliation, applying the traditional consotiational Lebanese model. Moreover, there are other non-state actors keeping important weaponry, although some of them (as in the case of Hezbollah) cooperate with the LAF in a very peculiar model of governance. Since the withdrawal of the Syrian troops in 2005, the country is split concerning how to tackle a security sector reform. While some actors only wanted to preserve the status quo (with Syrian and Iranian support), other only want to disarm Hezbollah (backed by the US and Saudi Arabia). The objective of this paper consists of assessing the degree to which the past reforms have contributed to the creation of a security sector adapted to the requirements of a democratic system and able to guarantee domestic peace, political neutrality and national defence.
\end{abstract}

Keywords: security sector reform, Lebanon, consotiationalism, Lebanese Armed Forces, borders.

1 Departamento de Historia, Teorías y Geografía Políticas, Universidad Complutense. Correo: flion@ucm.es Este artículo se inscribe en el marco del proyecto de investigación dirigido por la Laura Feliú Martínez y Ferrán Izquierdo Brichs, «Dinámicas y actores transnacionales en Oriente Medio y Norte de África (MENA): una genealogía histórica de élites y movimientos sociales entre lo local y lo global» (Ministerio de Economía y Competitividad, Agencia Estatal de Investigación y Fondo Europeo de Desarrollo Regional) (HAR2016-77876-P) 
Sumario. 1. Introducción 2. Marco teórico 3. El sector de la seguridad en la I República libanesa. Consociacionalismo y colaboración público-privada 4. La II República libanesa. Desarme, desmovilización y reconciliación 5. La salida de las tropas sirias y el nuevo modelo de cooperación público-privado 6. Conclusiones. Bibliografía.

Cómo citar: Lion Bustillo, J., «Reforma del sector de la seguridad en el Líbano: Consociacionalismo, securitización o democratización», en Cuadernos de Gobierno y Administración Pública 6-1, 23-40.

\section{Introducción}

Si el Oriente Medio se caracteriza en general por la presencia de unos fuertes aparatos de seguridad bajo el rígido control de las autoridades, en el caso libanés podemos hablar de un modelo claramente peculiar y diferenciado, estrechamente vinculado con las características del sistema político consociacional existente en el país. Este modelo libanés se halla bastante alejado del concepto weberiano de un Estado poseedor del monopolio de la violencia legítima dentro de sus fronteras y de unas Fuerzas Armadas encargadas de la defensa de su soberanía frente a cualquier amenaza exterior. Muy al contrario, los pactos que dieron origen al surgimiento de la I República libanesa se basaban sobre todo en el temor de los distintos actores a que las nuevas instituciones estatales acumularan una excesiva cantidad de poder en sus manos, capacitándolas para desafiar el control que los hombres fuertes del país (zu'ama) ejercían sobre sus respectivas comunidades confesionales. Esto se reflejó en todas las políticas públicas, caracterizadas por dos tendencias fundamentales: el intento de captura de las instituciones por parte de los partidos, estableciendo cuotas de reclutamiento confesional, y el establecimiento de mecanismos de colaboración públicoprivada que limitaran la capacidad de esas mismas instituciones en el ejercicio de su propia autonomía de acción política (Fakhoury Mühlbacher, 2007: 77-80).

Con la victoria siria en la guerra civil, los Acuerdos de Paz de Ta'if (1989) permitieron a Damasco llevar a cabo una reforma del sector de la seguridad que supuso la pervivencia de múltiples agencias, pero puestas bajo el rígido control sirio gracias a la presencia de numerosas unidades militares y agentes de inteligencia de ese país. En este contexto, las milicias de los partidos fueron oficialmente disueltas, si bien conservaron ciertas estructuras mínimas (varios cientos de hombres en cada una de ellas, dotados de armamento ligero). La excepción fue Hezbollah, ya que esta milicia chií jugaba un papel importante al garantizar el hostigamiento a las Fuerzas de Defensa de Israel y a sus aliados en la denominada "zona de seguridad" del Sur del país (Picard, 1999).

Tras la llamada "Primavera del Cedro" (2005), las tropas sirias tuvieron que abandonar el Líbano, de manera que fue necesaria una nueva reforma del sector de la seguridad. Sin embargo, el clima de división en el país y las desconfianzas mutuas han conducido a que la misma haya quedado en buena medida bloqueada. Mientras las FAL han experimentado una cierta reconstrucción y han recuperado buena parte de su prestigio para un buen número de libaneses, la proliferación y captura de las agencias de seguridad por parte de los diferentes partidos ha continuado, resultando muy difícil el llevar a cabo una política coherente y el evitar su instrumentalización partidista (Nerguizian, 2009: 11). Por otro lado, ha resurgido la vieja tendencia a la colaboración público-privada en la gestión de la seguridad, ya que mientras Hezbollah ha manteni- 
do (y mejorado) su componente militar, desarrollando una cooperación discreta con las FAL en la lucha contra el yihadismo salafista, otros partidos han reforzado sus milicias privadas. Todo esto nos permite hablar de que el Líbano es un Estado caracterizado por un modelo de gobernanza del sector de la seguridad peculiar en su región, en el cual el carácter consociacional del régimen político y el fuerte papel de las comunidades confesionales se han reflejado en plenamente en el funcionamiento de las instituciones y agencias de seguridad. Éstas han tenido serias dificultades a la hora de llevar a cabo una acción concertada en el cumplimiento de sus atribuciones, dándose más bien una competencia entre ellas. La única excepción han sido las Fuerzas Armadas, que han experimentado una evolución histórica a favor de una mayor representatividad social e integración nacional (Barak, 2009). Por otra parte, la pervivencia de una cierta colaboración entre lo público y lo privado ha sido una característica permanente del sector de la seguridad libanés a lo largo del tiempo, por lo que poner fin a esta situación no resultará sencillo en absoluto, dada la escasa confianza existente entre los distintos partidos libaneses y su reticencia a que se creen instituciones estatales fuertes que puedan minar el control social ejercido por los líderes tradicionales de las respectivas comunidades (Van Veer, 2015). En cualquier caso, si el país pretende avanzar en el camino de la democratización, será necesaria una reforma en profundidad del sector de la seguridad que garantice el imperio de la ley y evite que sirva a los fines particulares de los distintos líderes y partidos.

La estructura de este trabajo es la siguiente: en la primera parte se hace referencia a las principales fuentes del marco teórico que va a ser empleado; en la segunda, se exponen las características históricas de la gobernanza del sector de la seguridad en la I República libanesa y el impacto sobre la misma de la guerra civil; a continuación, se analizarán los problemas para la reforma del sector de la seguridad en la II República, especialmente en lo relativo al desarme de las milicias; posteriormente, se analizará el impacto de la retirada siria y de la guerra civil en el vecino país; por último, se extraerán algunas conclusiones sobre la especificidad del modelo libanés de seguridad y su posible reforma.

\section{Marco teórico}

El concepto de gobernanza del sector de la seguridad y de su reforma pone el acento en la cuestión de la implicación de los diferentes actores sociales en la misma, estableciendo un lazo con la democratización de un país y con la contribución que a la misma pueden hacer las distintas ramas del sector de la seguridad. Además, toca la cuestión del monopolio de la violencia legítima, en términos weberianos, y la de hasta qué punto dicho monopolio puede estar minado en la actualidad por la tendencia a la privatización del mismo debido a factores como el impacto de la globalización o la creciente implicación de la comunidad internacional en aspectos relacionados con la seguridad (por ejemplo, la intervención de actores internacionales en escenarios post-conflicto). Así, mientas algunos autores se han referido a estos casos como "Estados frágiles", otros han desarrollado el concepto de regímenes híbridos, entendiendo por tales aquéllos en los cuales hay una coexistencia de formas de autoridad estatales y no estatales (Boege et al., 2009). En este contexto se produce tanto la proliferación de actores subestatales que emplean la fuerza como la de agencias privadas en las que el Estado delega algunas actividades de seguridad (Bryden, 2006; 
Wulf, 2007). De ahí que se empleen conceptos como "paz híbrida" y "gobernanza de seguridad híbrida” (Richmond, 2015; Schroeder et al., 2014).

Los estudios sobre el sector de la seguridad en el Líbano son bastante más abundantes que los existentes sobre este tema en otros países árabes, lo que sin duda se encuentra influido por un factor decisivo: el hecho de que el Líbano sea un país plural con altos niveles de libertad de expresión y con una vibrante investigación académica. Sin embargo, cabe notar una gran desigualdad, ya que mientras la mayoría de los trabajos se han centrado en el papel de las FAL, el resto de las agencias ha recibido mucha menor atención. Así, hay estudios que han abordado principalmente la evolución de la composición sociológica de las FAL desde su fundación hasta la actualidad, incluyendo aspectos como su procedencia confesional, social, de clan o geográfica. También se ha puesto el énfasis en su ideología, su visión de la nación o su percepción de la seguridad, así como el papel que esta institución ha desempeñado desde la guerra civil hasta la actualidad (Kechichian, 1985; Sigaud, 1988; Mc Laurin, 1991; Barak, 2006; Gaub, 2007).

Tras la "Primavera del Cedro" (2005) se han publicado distintos trabajos centrados en el desarrollo global de una reforma del sector de la seguridad con vistas a consolidar los cambios en el sistema político (Belloncle, 2006; Sayigh, 2009). Por su parte, otros estudios han abordado la reforma de instituciones y agencias específicas, tales como las FAL o las Fuerzas de Seguridad Interior (FSI) (Nerguizian, 2009). El principal problema es que algunas de las iniciativas de reforma se centran simplemente en cómo lograr un fortalecimiento de la posición política de alguna de las dos coaliciones ( 8 y 14 de marzo) que se disputan el poder, sin prestar demasiada atención a cómo construir un sector de la seguridad que proteja la soberanía nacional, los derechos humanos y las libertades, garantizando el imperio de la ley. En este sentido, las propuestas técnicas de reforma se mezclan, por tanto, con los intereses de la confrontación política. En otras palabras, en ocasiones la reforma del sector de la seguridad puede ser utilizada no para reforzar el control democrático del aparato de seguridad, sino para fortalecer su capacidad de combate frente a un enemigo determinado, adoptando una perspectiva de securitización (Wulf, 2011: 340-2).

Por último, hay que destacar los estudios dedicados al papel que los distintos actores internacionales han jugado en el sector de la seguridad libanés. No podemos olvidar que desde 1978 existe una fuerza de Naciones Unidas (la Fuerza Interina de Naciones Unidas para el Líbano, UNIFIL) desplegada en el Sur del país, tratando de evitar enfrentamientos con Israel. Además, ha sido frecuente la presencia de tropas de distintas nacionalidades (israelíes, sirias, norteamericanas, francesas...). Todos estos actores han desarrollado un tejido de relaciones más o menos complejas con las instituciones y los grupos políticos libaneses, de manera que su influencia ha sido notable en la evolución del conflicto. En esta área, el caudal de estudios es muy abundante, destacándose que mientras los contingentes de Naciones Unidas han mantenido una presencia difícil pero tolerada por los actores libaneses, los contingentes puramente nacionales han experimentado serias dificultades a la hora de legitimar su papel, lo que a la larga ha conducido a su retirada en circunstancias complejas. En cualquier caso, la presencia de la FINUL habría propiciado el surgimiento de un modelo híbrido de seguridad caracterizado por la colaboración a largo plazo entre estas fuerzas y los actores locales (Newby, 2016). Al mismo tiempo, otros actores internacionales han tratado de influir en la reforma del sector de la seguridad en el Líbano mediante programas de ayuda y colaboración (Tholens, 2017). 
El concepto de securitización fue lanzado hace varias décadas por autores pertenecientes a la Escuela de Copenhague, aplicándose a un proceso por el cual en primer lugar se identifica a nivel discursivo la existencia de una amenaza de carácter existencial para cualquier bien considerado como digno de ser preservado por una comunidad. En otras palabras, este movimiento de securitización implicaría la construcción social de una amenaza ante una audiencia, para lo que sería necesaria una cierta capacidad de poder e influencia. El segundo paso implicaría propiamente la adopción de medidas excepcionales justificadas por la existencia de dicha amenaza, con lo que un determinado tema abandonaría la esfera de la política y pasaría a tratarse plenamente dentro de la esfera de la seguridad, con el excepcionalismo que ello implicaría (Waever, 1995; Taureck, 2006).

En el caso del Líbano, los distintos grupos políticos tienden a hacer uso de los mecanismos de securitización como un medio para mantener unida a su base política y para impedir cambios que resulten perjudiciales para sus intereses particulares. A pesar de que la guerra civil concluyó hace casi treinta años, la desconfianza entre grupos políticos y entre comunidades sigue siendo notable, lo que refuerza la posición de preeminencia de los actuales partidos confesionales (consolidados durante la guerra civil gracias a su papel militar) y evita el surgimiento de alternativas interconfesionales que promuevan reformas profundas del sistema político y de la Administración Pública. Igualmente, la actuación de las distintas potencias ha reforzado esta estrategia de securitización, centrando su actuación en extender su propia influencia en las estructuras político-administrativas del país en lugar de buscar su independencia y servicio a los intereses nacionales (Fakhoury Mühlbacher, 2007: 391-418).

\section{El sector de la seguridad en la I República libanesa. Consociacionalismo y colaboración público-privada}

El sector de la seguridad en el Líbano ha presentado unas características históricas estrechamente vinculadas al particular modelo político existente en el país. Así, la estructura consociacional condujo a que las distintas instituciones con responsabilidades en el terreno de la seguridad nacional trataran de ser controladas por los diferentes grupos políticos, al tiempo que su número proliferaba con el objetivo de que cada grupo tuviera su propia agencia. No obstante, el papel hegemónico de los partidos cristianos maronitas en la política del país se reflejó en su control de las principales instituciones de seguridad. Entre ellas, destacaba el papel de las Fuerzas Armadas (FAL), las cuales presentaban una enorme debilidad en cuanto a medios y personal con vistas a garantizar la soberanía nacional en un territorio tan convulso como el Oriente Medio tras la II Guerra Mundial. Precisamente las FAL nacieron en 1945, viéndose rápidamente envueltas en la guerra árabe-israelí de 1948-49, si bien su actuación resultó muy limitada. A partir de ese momento, su labor se centró por completo en la preservación del orden interior, tratando de mantenerse al margen de los conflictos existentes entre gobierno y oposición, evitando ver comprometida su imagen de neutralidad. Un ejemplo muy claro lo constituyó el no participar en los combates entre partidarios del Presidente Camille Chamoun y los de la oposición en 1958. Esta actitud tendente a evitar tomar partido ha garantizado a las FAL un destacado papel protagonista en política, no mediante la habitual fórmula en el Oriente Medio del golpe de Estado, sino como opción intermedia que permite la superación 
de fases de falta de acuerdo en la designación de candidatos para ciertas magistraturas. La elección presidencial del general Antoine Chehab en 1958 constituyó así un precedente para otras situaciones similares (Gendzier, 2006). No obstante, el puesto de Comandante en Jefe de las FAL recaía necesariamente en un cristiano maronita, siendo principalmente personas de esta confesión las que dominaban la jerarquía militar, ya que los suníes no estimaron demasiado este tipo de salida profesional, mientras que en los chí́es su peor formación educativa constituyó un obstáculo para ingresar en la Academia Militar, a lo que hay que añadir factores relacionados con el capital social de cada individuo, como la pertenencia a determinadas familias, clanes, regiones geográficas, que favorecían las posibilidades de ser reclutado entre la oficialidad. Este predominio maronita creaba una tensión entre la defensa de una identidad nacional libanesa y la influencia de una identidad maronita que veía a los integrantes de esta comunidad como los líderes naturales del Estado libanés.

Si la oficialidad era predominantemente maronita, buena parte de la tropa que conformaba el Ejército se hallaba compuesta de musulmanes suníes y chíies, los cuales desconfiaban de las preferencias políticas de sus oficiales. Además, el Ejército se agrupaba en un total de doce brigadas, caracterizadas por su composición confesional y por su procedencia geográfica unificada, existiendo por ejemplo una Brigada drusa de las Montañas. En otras palabras, las FAL vivían en un equilibrio inestable que trató de ser contenido por Antoine Chehab mediante la promoción de un nacionalismo libanés que sin desafiar el statu quo permitiera al mismo tiempo crear un Estado más abierto e integrador. Específicamente, las FAL fueron dotadas de un proceso de reclutamiento en el que se ponía mayor énfasis en respetar las cuotas demográficas de las respectivas comunidades, además de favorecer una procedencia geográfica y social más abierta (Barak, 2006: 79-80).

El fenómeno del chehabismo hizo sin embargo sonar las alarmas entre la élite maronita, ya que favoreció una concentración de poder que desafiaba los tradicionales equilibrios existentes en el país. A ello también contribuía el papel político de la inteligencia militar (el denominado Deuxième Bureau), que desarrolló una actividad de discreta, pero constante intervención en la vida política del país. Por todo ello, la élite maronita optó en 1970 por cortar esta influencia militar con la elección presidencial de Suleiman Franjieh. El nuevo Presidente sucumbió a la tentación de emplear a las FAL en la represión de la oposición en los Años 70, además de tratar de cortar las actividades de los combatientes palestinos instalados en el país en los últimos años, para lo cual las FAL desarrollaron una estrecha colaboración con las milicias maronitas. En ese momento, por un lado, el Estado libanés experimentó una clara pérdida de su monopolio sobre la violencia legítima, quedando ésta repartida entre diversos actores. Por otro, las FAL perdían su imagen de neutralidad y se acercaban cada vez más a los intereses de los partidos maronitas, agrupados en el Frente Nacional. La consecuencia de este último factor fue la propia fragmentación de las FAL, dando origen a una escisión (el denominado Ejército Árabe del Líbano) en 1976 y a la posterior integración de muchos de sus efectivos en las distintas milicias (Barak, 2009: 100-109).

Lo que quedó de las FAL pasó a ser simplemente un instrumento al servicio del Partido Falangista tras la elección de Amin Gemayel como Presidente de la República en 1982, participando activamente en la lucha contra las milicias opositoras. Sin embargo, en 1988 las disputas internas entre los líderes maronitas condujeron al hasta entonces Comandante en Jefe de las FAL, general Michel Aoun, a combatir 
contra las Fuerzas Libanesas de Samir Geagea en una lucha que concluyó con la victoria siria en la guerra civil y la aprobación de los Acuerdos de Ta'if (1989). Durante la guerra civil, la privatización del sector de la seguridad fue casi total, con unas milicias que se repartieron el control territorial del país y que establecieron enclaves en los que desarrollaban las funciones habituales de un Estado, incluyendo el mantenimiento del orden público y la defensa del territorio frente a desafíos exteriores, si bien buena parte de sus energías las dedicaron a derrotar a otras milicias rivales pertenecientes a su misma confesión, marginando completamente a las FAL (Picard, 1999: 3).

La principal fuerza policial del Líbano son las Fuerzas de Seguridad Interior (FSI), creadas ya en el siglo XIX y que experimentaron un importante desarrollo durante el Mandato francés, manteniendo un carácter de fuerza paramilitar, al dotarse de un armamento apreciable y poseer una formación obtenida igualmente en la Academia Militar. Su composición reflejó claramente un predominio suní (bajo el control del Primer Ministro), compensando así el predominio cristiano en las FAL. Las FSI demostraron serias dificultades a la hora de adaptarse al cumplimiento de sus funciones, a lo que no ayudaba en absoluto el fuerte fraccionamiento de las actividades policiales entre distintas agencias. En 1953 se creó mediante el decreto legislativo 1953-59 la Dirección General de las FSI, mientras que en 1959 surgía la Dirección General de Seguridad General (SG) mediante el decreto legislativo n $^{\circ} 139$, que se ocupaba especialmente de la recogida de información en la lucha contra las actividades delictivas, además de desempeñar las cuestiones relativas a las fronteras, puertos y aeropuertos. El papel de estas agencias de seguridad quedó reducido casi a la nada durante la guerra civil, ya que las milicias se hicieron con el control del orden público en sus respectivos enclaves, completando así la tendencia hacia la privatización del sector de la seguridad en el país (Saliba, 2012: 16-7).

\section{La II República libanesa. Desarme, desmovilización y reconciliación}

Con la victoria militar siria y los Acuerdos de Paz de Ta'if, el sector de la seguridad libanés quedó reconstruido a partir de los intereses sirios, pero sin desafiar las tradiciones del modelo consociacional libanés. Muy al contrario, Hafez al-Assad supo utilizar algunas de las características de ese modelo claramente en su favor, ya que le podría permitir reforzar su control sobre el país vecino. El final de la guerra supuso el desarme (voluntario o por la fuerza) de las distintas milicias. Algunas de ellas entregaron las armas a cambio de un puesto destacado en la política nacional y de que sus líderes mantuvieran las fortunas amasadas durante el conflicto. Una parte de los milicianos pasó a integrarse en las FAL o en otras agencias de seguridad, mientras otros buscaron su retorno a la vida civil (Picard 1999).

Sin embargo, no se puede hablar de un proceso de desarme total, ya que las distintas milicias mantuvieron algunos combatientes (normalmente, varios centenares), así como cierto armamento ligero, si bien evitaron el mostrarlo en público. Por otro lado, Hezbollah quedó exenta de ese desarme, ya que la ocupación israelí del Líbano meridional pasó a convertirse en la justificación aducida para ello. De hecho, Hezbollah se convirtió en un instrumento muy útil para Damasco, ya que sus operaciones minaban la ocupación israelí en el Sur, lo que pretendía convencer a Tel Aviv de la necesidad de negociar con Assad la suerte del Golán. Por ello, Hezbollah pasó a re- 
cibir armamento sirio siempre y cuando el mismo fuera utilizado contra Israel (Siegman, 2000). De hecho, la milicia chí mantuvo durante esta etapa este compromiso, lo que fomentó su imagen de personificación de la resistencia libanesa frente a Israel, algo reconocido por los diferentes gobiernos libaneses bajo la fórmula "Ejército, Pueblo y Resistencia". Como plasmación de este pacto entre Damasco y Hezbollah, el Ejército libanés no se desplegó al Sur del Litani, mientras que los choques entre Hezbollah y las tropas israelíes se repitieron con cierta frecuencia, estableciéndose entre ellos un "equilibrio de mutua disuasión" basado en unas reglas del juego que han cambiado a lo largo del tiempo (Malka, 2008).

Otro factor a tener en cuenta fue que, en los campamentos palestinos, las milicias de la OLP conservaron su armamento, de tal forma que la seguridad dentro de los mismos continuó quedando en sus manos. El problema fue, por un lado, la existencia de disputas internas dentro de las propias organizaciones integradas en la OLP (algunas de ellas bajo una clara influencia siria), mientras que, por otro, el liderazgo de la propia OLP entre los refugiados comenzó a experimentar un evidente declive a medida que el proceso de paz de Oslo fue mostrando sus limitaciones, especialmente la de no dar una respuesta a la petición de derecho de retorno a Palestina. Esto provocó el surgimiento de fuerzas alternativas (especialmente Hamas) que rechazaban los acuerdos de Oslo y que adoptaban una ideología islamista en su lucha contra la ocupación israelí. Pronto en los campamentos de refugiados aparecieron algunos de estos grupos, siendo destacable la presencia de organizaciones cercanas al yihadismo salafista internacional, las cuales no focalizaban su atención en la lucha contra Israel, sino que pretendían un doble objetivo: la islamización de las costumbres en los campos de refugiados y la participación en cualquier lucha a escala mundial que apoyara una Yihad global. En otras palabras, mientras el Estado libanés ignoraba en buena medida las necesidades de los refugiados palestinos, envueltos en un estado de permanente marginación, los campos de refugiados pasaban a convertirse en centros de formación y reclutamiento de militantes yihadistas salafistas, desarrollando fuertes vínculos de cooperación con organizaciones libanesas de la misma ideología que aprovechaban el vacío de seguridad de los campos y el apoyo de algunos clérigos para robustecer su presencia en territorio libanés y para participar en acciones violentas en otros escenarios (Irak, Chechenia, Afganistán...). Cuando dichos grupos trataron de desafiar al Estado, el resultado fue una confrontación directa con las FAL, como se puso en evidencia en la región de Akkar con el denominado "grupo Dinniyeh" en 1999 (Rougier, 2015) ${ }^{2}$.

Con respecto a las FSI, éstas experimentaron una transformación muy importante, ejerciendo Damasco un enorme control sobre ellas. Las mismas estaban integradas principalmente por policías suníes (proporcionando puestos de trabajo para integrantes de esa comunidad cercanos a los políticos suníes que colaboraban con Siria). Por otra parte, sus funciones no quedaron claramente separadas de las del Ejército. Por el contrario, dada la fuerte implicación de éste en el mantenimiento del orden público, las FSI pasaron a convertirse en una especia de fuerza auxiliar en el desempeño de esta labor. Al propio tiempo, el decreto 1157 de 1991 creó una Sección de Información, la cual adoptó las características de una agencia de inteligencia diferen-

2 Majmouat al-Dinniyeh fue un grupo yihadista vinculado a Al Qaeda que lanzó un ataque contra las FAL en 1999, siendo posteriormente desarticulado (Lion Bustillo, 2017: 109). 
ciada del Deuxième Bureau del Ejército y de la Seguridad General. Durante el mandato presidencial de Émile Lahoud, se adoptó la decisión de que la Seguridad General, hasta entonces dirigida por un cristiano, pasara a manos chíes, a cambio de que los cristianos recibieran el control de la denominada Seguridad del Estado, una agencia creada en 1983 por Amin Gemayel. Posteriormente, esta agencia ha sido oficialmente suprimida, aunque ha continuado su existencia de forma más o menos discreta. La Seguridad General se ocupaba fundamentalmente de aspectos vinculados con la información, destacando la cuestión de las relaciones con los refugiados palestinos, un tema de especial sensibilidad dada la presencia entre estos últimos de distintas facciones armadas (Gaub, 2007; Saliba, 2012: 14).

La principal característica de estas agencias es que servían para el propósito sirio de aplicar el principio de "divide y vencerás", promoviendo una cierta competencia entre las mismas con vistas a eludir cualquier fuerte resistencia a la hegemonía de Damasco, al tiempo que se disputaban entre sí el favor de Hafez al-Assad. Además de poseer un personal de enlace en estas agencias, la propia inteligencia siria se hallaba desplegada en territorio libanés, lo que le permitió un rígido control de cualquier amenaza. Por otro lado, el régimen de Assad desarrolló una importante labor de socialización entre la élite de seguridad libanesa, la cual recibía habitualmente parte de su formación en la propia Siria, consiguiendo incluso el apoyo norteamericano para estas actividades (Ziadeh, 2011: 80-81).

\section{La salida de las tropas sirias y el nuevo modelo de cooperación público- privado}

Sin embargo, la denominada "Primavera del Cedro" puso en evidencia las dificultades que las fuerzas de Assad tenían para controlar el país vecino, coincidiendo además con una fuerte campaña de presión internacional para que Damasco retirara sus tropas y agentes de seguridad, la cual se completó en 2005. Con la llegada al poder de un gobierno mayoritariamente hacia Siria dirigido por Fouad Siniora ${ }^{3}$, las circunstancias experimentaron cambios significativos. Las FSI pasaron a convertirse en el instrumento predilecto del gobierno, dada la clara hegemonía de la familia Hariri y su Movimiento de Futuro entre la comunidad suní y su enorme desconfianza hacia otras agencias de seguridad. En este sentido, su rama de inteligencia constituyó el instrumento básico de recogida de información para las nuevas autoridades, al tiempo que sus agentes pasaron a convertirse en la principal fuerza de choque para el gobierno Siniora en el caso de que la crisis política existente desembocara en un conflicto civil abierto. Abundando en esta tendencia, distintas potencias aliadas del Movimiento de Futuro (Estados Unidos, Arabia Saudí) aportaron importantes recur-

\footnotetext{
El nuevo gobierno libanés estaba mayoritariamente compuesto por miembros de la Coalición 14 de marzo, que incluía a partidos como el Movimiento de Futuro de la familia Hariri, las Fuerzas Libanesas, la Falange o el Partido Socialista Progresista. Pero también estaban presentes algunos integrantes de la Coalición rival, el 8 de marzo, compuesta principalmente por Hezbollah y Amal. El posterior compromiso entre Hezbollah y el mayor partido cristiano, el Movimiento Patriótico Libre, ha creado un equilibrio de poder entre ambas coaliciones, de tal manera que el resultado han sido unos gobiernos de concentración nacional en los que la toma de decisiones resulta compleja.
} 
sos para reforzar a las FSI y convertirlas en una milicia paramilitar capaz de enfrentarse a Hezbollah si ello fuera necesario (Nashabe, 2009: 9; Sayigh, 2009: 16-17).

Por estas mismas razones, Irán y Siria estrecharon su colaboración con Hezbo1lah, Amal y otros grupos cercanos a Damasco, ya que temían que, una vez las fuerzas sirias se habían retirado del Líbano, existía un riesgo de que las nuevas autoridades adoptaran una política que minase su influencia. En esta labor, el control de ciertas agencias de seguridad resultaba clave, por ejemplo, la Seguridad General y el Servicio de Seguridad del aeropuerto de Beirut, el cual posee gran importancia en lo relativo a garantizar envíos de armas u otros recursos. Igualmente, resultaba muy importante el mantener el monopolio de la fuerza al Sur del río Litani, de modo que en la frontera con Israel sólo hubiera combatientes de Hezbollah. Esto permitía una enorme flexibilidad en sus actividades sin ningún tipo de control gubernamental y una capacidad absoluta de implementar su estrategia de disuasión de forma unilateral, sin responder ante las autoridades. En otras palabras, Hezbollah asumía así una función típicamente estatal como es la del control de las fronteras y la de disuasión ante los enemigos exteriores. El gobierno Siniora exigió repetidamente el poner fin a esta situación mediante el desarme de Hezbollah y el despliegue de tropas de las FAL al Sur del Litani. Sin embargo, esta posibilidad quedaba descartada no sólo por el rechazo de la milicia chií, sino también por el del Presidente de la República, Émile Lahoud ${ }^{4}$.

Desde la retirada israelí del Líbano meridional en el año 2000, Hezbollah (y las propias autoridades libanesas) han afirmado que dicho movimiento fue incompleto, considerando que el área de las Granjas de Shebaa constituía un territorio libanés todavía bajo ocupación. Por ello, las operaciones bélicas puntuales continuaron entre ambas partes, desembocando en la Guerra de 2006, en la cual durante más de un mes de combates las tropas israelíes infligieron cuantiosos daños a la milicia de Hezbollah y al conjunto del país, pero sin lograr una victoria decisiva. La guerra tuvo un doble efecto en el país, ya que mientras muchos criticaban un comportamiento excesivamente aventurero por parte de Hezbollah, también se consideraba por parte de numerosos ciudadanos que la magnitud de la represalia israelí y la fortaleza de la resistencia habían demostrado que el Líbano debía conservar una fuerza de disuasión ante un vecino poco respetuoso con la soberanía libanesa. Durante el conflicto, las FAL se abstuvieron de intervenir en los combates, pero ciertas evidencias sugieren que colaboraron con Hezbollah en el intercambio de información, lo cual fue percibido en Washington como una señal de que esta institución no resultaba fiable (Sayigh, 2009: 9).

Esto nos conduce a la cuestión del complejo y delicado equilibrio de acción de las FAL en un momento de rígida división social y política del país. Las FAL han basado siempre su prestigio en su carácter nacional y no partidista, de manera que un apoyo al gobierno Siniora habría sido percibido como un alejamiento de esa identidad nacional, comportándose como otras agencias de seguridad. Por el contrario, ignorar al nuevo gobierno parecía alejarse de la opinión pública, buena parte de la cual se había mostrado muy crítica con la presencia siria. Entre los mandos de las FAL existe una importante presencia cristiana, teniendo un gran peso el legado del general Fouad

4 Una zona clave en la colaboración entre Siria y Hezbollah era la frontera entre ambos países, la cual quedaba claramente fuera del control efectivo de las autoridades de Beirut (Harris, 2007). 
Chehab y su visión integradora y nacionalista. En este sentido, muchos de estos oficiales rechazan la idea de alinearse con ningún partido en un enfrentamiento armado, prefiriendo mantenerse al margen de esas confrontaciones. De ahí que, en los momentos de mayor división interna, las FAL sean reticentes a tomar partido en cualquier sentido. Este mismo factor fue el que provocó que en el gobierno Siniora las FAL fueran vistas como un elemento con el que no se podía contar, prefiriendo que la ayuda armamentística exterior se concentrara en las FSI (Narguizian, 2009: 1011).

Las autoridades estadounidenses han tenido en cuenta estos mismos factores en su política hacia el sector de la seguridad libanés, concentrando su ayuda en aquellas instituciones cercanas al 14 de marzo e ignorando al resto, buscando así alterar el equilibrio de poder en el propio sector y en la política nacional. Desde la Revolución del Cedro se había desarrollado un programa de ayuda norteamericana a las FAL con el objetivo de que en el futuro llegaran a enfrentarse militarmente a Hezbollah. Sin embargo, las reticencias de las FAL a jugar ese papel y la colaboración de su Departamento de Inteligencia con Hezbollah durante la guerra de 2006 contra Israel sembraron el descontento de la Administración Bush. A ello hay que añadir que Israel ha actuado a menudo como actor con poder de veto ante cualquier mejora significativa de las capacidades militares de las FAL que les permitan un incremento de su poder de disuasión (misiles tierra-aire, misiles antitanques...) (Narguizian, 2009: 26-29). Curiosamente, este factor mantiene a las FAL en una posición de debilidad, lo que refuerza el argumento de Hezbollah de que sus recursos son necesarios para garantizar una capacidad de disuasión libanesa frente a cualquier vulneración de su soberanía. A partir del verano de 2006, a pesar del despliegue de las FAL en el Sur del país (en cumplimiento de la Resolución 1701 del Consejo de Seguridad, impulsada por Washington), Estados Unidos decidió que la agencia de seguridad que pasaría a recibir el grueso de su ayuda serían las FSI, marginando claramente a las FAL y privándoles de unos recursos imprescindibles para desarrollar su labor (Sayigh, 2009: 8-9). Curiosamente, no hubo una intención clara de mejorar la actuación de las FSI en materia de defensa de derechos humanos e imperio de la ley, áreas en las que la reforma habría sido menos controvertida y habría contado con amplios apoyos en la sociedad civil (El Mufti, 2015: 5).

Las FAL centraron su atención en la lucha contra el yihadismo internacional, cuyo crecimiento se ha visto también favorecido por la pobreza general existente, a lo que hay que unir la presencia de los campos de refugiados palestinos, en los que esa radicalización ha resultado evidente ante la falta de resultados del proceso de paz árabe-israelí y la situación de marginación en la que vive esa comunidad en suelo libanés. El campo de refugiados de Nahr el-Bared, en las cercanías de Trípoli, fue el escenario en 2007 del surgimiento de una organización, Fatah al-Islam, decidida a extender la acción yihadista contra Estados Unidos y Hezbollah, lo que le llevó a un enfrentamiento directo con las FAL que se saldó con una elevadísima cifra de víctimas en ambos bandos y entre la población civil. Desde entonces, este y otros campos palestinos han proporcionado combatientes para los grupos del yihadismo salafista a escala regional (Rougier, 2015: 82-170).

Las tensiones sobre el control del sector de la seguridad se pusieron en evidencia tras la crisis de gobierno en 2007 y el abandono del mismo por parte de los ministros cercanos al 8 de marzo (lo que incluyó al conjunto de los chiíes, cuestionando el cumplimiento del Pacto Nacional). Las decisiones del gobierno Siniora de mayo de 
2008 por las que ponía bajo su autoridad la red telefónica de comunicación interna de Hezbollah y el Servicio de Seguridad del aeropuerto desembocaron en la llegada a Beirut Oeste de milicianos de Hezbollah, Amal y del Partido Socialista Nacional Sirio, los cuales se enfrentaron con combatientes del Movimiento de Futuro y del Partido Socialista Popular druso por el control de los barrios clave de las fuerzas gubernamentales. Las distintas agencias de seguridad optaron por mantenerse al margen de la lucha, ya que su intervención habría podido desembocar en un enfrentamiento generalizado (Narguizian, 2009: 18-19). En este sentido, el papel del Comandante en Jefe de las FAL, el general Michel Suleiman, siguió los patrones tradicionales del chehabismo, garantizando la neutralidad de la institución y evitando su uso con fines partidistas. En este contexto, la victoria de las milicias del 8 de marzo fue nítida en Beirut, si bien tanto en el Chouf como en Trípoli el equilibrio de fuerzas fue diferente. Los líderes del 14 de marzo optaron por reconocer la superioridad militar de Hezbollah y esto implicó el rápido fin de los combates. A su vez, los milicianos chíes evitaron provocaciones y fueron retirados inmediatamente, dándose paso a una negociación bajo mediación qatarí. El resultado final, los Acuerdos de Doha, reconoció que un tercio del gobierno podía bloquear cualquier iniciativa propuesta, lo que satisfacía los intereses del 8 de marzo, ya que ello garantizaba que no habría ninguna reforma significativa del sector de la seguridad que no estuviera basada en el consenso. A su vez, esta misma minoría de bloqueo era una garantía para el propio 14 de marzo para un futuro en el que sus rivales ostentaran la mayoría parlamentaria (tal como ocurrió poco tiempo después). En otras palabras, el statu quo prevalecería, evitando trastocar de manera significativa los equilibrios de poder.

La guerra civil siria ha tenido un impacto muy profundo en la evolución del sector de la seguridad, ya que la confrontación armada en el país vecino se ha filtrado dentro del propio territorio libanés, dándose por parte de distintos grupos una acción de colaboración con sus aliados al otro lado de la frontera. Todas estas actividades poseían un carácter ilegal, desafiando el control que las autoridades de Beirut ejercían en teoría sobre las fronteras. Sin embargo, quienes realizaban dichas acciones eran a menudo miembros de partidos integrados en el propio gobierno y en las instituciones. En otras palabras, a pesar de que la denominada Declaración de Baabda (2012) expresó la neutralidad y la disociación del Líbano frente a la guerra en Siria, las fuerzas políticas libanesas no eran en absoluto neutrales y trataron de colaborar con aquellos actores sirios más útiles para sus intereses (Salem, 2012: 12-14; Lion Bustillo, 2014: 218-225).

Inicialmente, esta intervención tuvo un carácter discreto, basado en la transferencia de armas, combatientes y dinero a través de las fronteras, además de labores de entrenamiento en suelo libanés para combatientes sirios. Estas actividades eran desarrolladas tanto por parte de aliados de Bashar al Assad (como Hezbollah) como de la oposición (el Movimiento de Futuro, grupos yihadistas). Sin embargo, la escalada de la crisis siria y la amenaza directa de derrocamiento del régimen baasista condujo a Hezbollah a desempeñar un papel mucho más activo, enviando unidades de combate y asumiendo un papel protagonista en el conflicto sirio. Dentro de este nuevo papel, cabe destacar la labor de control de la frontera con vistas a evitar el uso de la misma por parte de milicias opositoras sirias situadas en la zona de las Montañas Qalamoun, las cuales se infiltraban a menudo en territorio libanés con el objetivo de descansar, rearmarse y retornar a Siria para proseguir sus acciones. El área de la ciudad de Arsal se convirtió en el espacio predilecto para esta clase de actividades, 
donde una población de mayoría suní era percibida como un aliado natural de los combatientes yihadistas pertenecientes a organizaciones como el Estado Islámico y Jabhat al-Nusra. De hecho, desde esta zona los combatientes yihadistas comenzaron a lanzar atentados contra miembros de Hezbollah, de la comunidad chií y contra las propias FAL, a las que acusaban de colaborar con Hezbollah en el cierre de la frontera. Por su parte, esta última milicia comenzó a tender emboscadas a ambos lados de la línea divisoria, en tanto que las FAL se centraban en su vigilancia. Esta división del trabajo aportó resultados muy positivos a la hora de prevenir nuevas infiltraciones y de debilitar la presencia yihadista en el Valle de la Bekaa, pero el precio a pagar ha sido un importante descontento entre la población suní de la zona que considera que las FAL han sido demasiado duras en la lucha contra el yihadismo salafista mientras que ignorarían las armas en manos de Hezbollah (Lion Bustillo, 2017: 121; International Crisis Group, 2016).

Otro espacio fronterizo en el que el impacto de la guerra en Siria ha sido muy destacado es la provincia de Akkar, donde una población suní enormemente empobrecida ha experimentado una fuerte radicalización, surgiendo diversos predicadores salafistas que han animado a ayudar a sus correligionarios sirios en su lucha contra el régimen de Assad, convirtiendo el conflicto sirio en una disputa confesional. El impacto de esta evolución ha sido muy negativo para el orden público, ya que han proliferado los grupos que han abrazado la bandera yihadista, tanto enviando combatientes a Siria como desarrollando ataques en suelo libanés, fundamentalmente contra las FAL y contra miembros de la minoría alauí, especialmente en la ciudad de Trípoli, en la que las tensiones intercomunales han alcanzado un punto muy elevado (Lefèvre, 2014). En esta zona, donde no hay combatientes de Hezbollah, los enfrentamientos se han dado entre militantes yihadistas contra los del Partido Democrático (de confesión alauí). Aquí el Ejército ha optado por una intervención activa, llevando a cabo importantes operaciones contra células yihadistas, en ocasiones vinculadas a Jabhat al-Nusra o al Estado Islámico, obteniendo importantes éxitos, si bien al precio de que su imagen se haya visto algo deteriorada en ciertos ámbitos suníes (Lion Bustillo, 2017: 122-124).

El cambio de signo en la guerra civil siria en favor de Bashar al Assad ha sido debido a distintos factores, pero cabe destacar que a partir de 2014 la frontera con el Líbano experimentó un creciente control por parte de las autoridades de ambos países y de la milicia de Hezbollah, a costa del debilitamiento del yihadismo salafista. En este sentido, cabe decir que en los últimos meses la posición de las FAL en las fronteras del país es mucho más fuerte de lo que ha sido durante el último medio siglo. Las fronteras Norte y Este han registrado el despliegue de los nuevos regimientos de infantería de fronteras, que además han visto facilitada su labor con la construcción de diversos puestos de observación y de bases de operaciones avanzadas, así como el levantamiento de numerosos puestos de control en dichas áreas (Nerguizian, 2017: 12-13). En este reforzamiento de sus capacidades, el papel de Estados Unidos y del Reino Unido ha sido clave, al aportar unos importantes recursos para la lucha contra el yihadismo trasnacional. Ello se tradujo en importantes éxitos en el verano de 2017, cuando una ofensiva de Hezbollah contra la zona fronteriza ocupada por Jabhat al-Nusra en las proximidades de Arsal fue seguida por un ataque de las FAL contra las defensas del Estado Islámico situadas algo más al Norte. El resultado global de la ofensiva ha sido la definitiva erradicación de ambas organizaciones del territorio libanés y la percepción de que las FAL se han convertido en una fuerza 
mucho más creíble en lo relativo a garantizar la soberanía nacional frente a la amenaza yihadista.

Y es que una de las características más destacables del sector de la seguridad en el Líbano en los últimos años ha sido el importante papel desempeñado por los programas de ayuda aportados de forma puramente bilateral por muy diversos países, que incluyen desde grandes potencias como Estados Unidos, Rusia y China, hasta potencias regionales como Arabia Saudí, a lo que hay que añadir el apoyo iraní a Hezbollah así como programas encubiertos de distintos países destinados a sostener las milicias de los diferentes partidos. La ayuda estadounidense ha estado estrechamente vinculada a la idea de lucha contra el terrorismo, dada la preocupante expansión del Estado Islámico en la región durante la guerra civil siria, poniendo un especial énfasis en asegurar un control territorial que impida esta expansión, si bien también un objetivo a más largo plazo sería el de dificultar la llegada de material militar para Hezbollah procedente de Irán (Tholens, 2017: 870-875).

Sin embargo, el hecho de que esta ayuda responda no a los intereses nacionales del Líbano, sino a los de las diferentes potencias implicadas, hace que en ocasiones se muestre un elevado grado de descontento ante la actuación de la Administración libanesa. Un ejemplo muy claro ha sido la postura de Arabia Saudí, prometiendo en 2014 una fuerte suma de dinero con vistas a modernizar las FAL con el supuesto propósito de contribuir al fortalecimiento de las instituciones y de la independencia del país. Este dinero estaría destinado a la compra de armamento francés, que serviría para modernizar el obsoleto equipo que poseen algunas unidades. Sin embargo, cuando el gobierno libanés decidió abstenerse en una condena de la Liga Árabe contra el régimen sirio, las autoridades saudíes anunciaron la retirada de dicha ayuda (Azakir, 2016). Un nuevo acto de intromisión saudí se dio cuando el Primer Ministro libanés Saad Hariri fue retenido en Riad y forzado a dimitir, para luego (ante las enormes protestas libanesas) ser autorizado a retornar y asumir de nuevo sus funciones. Al parecer, la causa de este incidente habría sido el disgusto de la monarquía saudí porque Hariri tendría un comportamiento excesivamente blando hacia Hezbo1lah (Barnard y Abi-Habib, 2017). En otras palabras, cualquier reforma del sector de la seguridad tendrá que tener en cuenta no solamente los equilibrios de poder nacionales, sino también las presiones de las distintas potencias, que desean cuidar de sus intereses en la región.

No obstante, el verdadero punto clave de la seguridad libanesa continuará estando en su frontera meridional, una vez que la guerra civil en Siria parece estabilizarse y el control del régimen de Damasco se ha reforzado en la zona occidental del país. Lo cierto es que estos últimos años han sido los más tranquilos en la zona desde finales de la década de los 60, ya que el nivel de enfrentamientos entre las Fuerzas de Defensa de Israel y Hezbollah ha sido muy bajo. Los incidentes más habituales han consistido en el descubrimiento a cargo de la FINUL o de las FAL de algunos depósitos de armas de la milicia chií al Sur del Litani. Por su parte, los israelíes han proseguido con las violaciones del espacio aéreo libanés. Sin embargo, los enfrentamientos directos han sido escasos. Un dato preocupante es que en ocasiones estos últimos han implicado a las tropas de las FAL, demostrando que su cercanía con las fuerzas israelíes y las tensiones entre ambos países hacían probable el estallido de estos incidentes. Por su parte, Hezbollah ha preferido mostrar un perfil bajo en la frontera con Israel en un momento en que sus recursos se hallaban muy extendidos en el vecino territorio sirio. 
Con el progresivo retorno de combatientes de Hezbollah y el gran debilitamiento del yihadismo salafista libanés, el equilibrio de fuerzas en el país ha cambiado, ya que precisamente la amenaza yihadista contribuyó en estos años a conformar un respaldo político sólido a las operaciones de las FAL contra esos grupos (Van Veer, 2015). Una vez superado esto, las FAL se encuentran en una posición compleja. Por un lado, existe una presión por parte de Washington y de Riad para que adopten una actitud cada vez más beligerante hacia Hezbollah, completando el control de las fronteras. En otras palabras, estos actores se centran en un modelo de reforma del sector de la seguridad basado en el paradigma de la securitización, de tal manera que dicha reforma debería centrarse en proporcionar a las FAL y a otras agencias de seguridad bajo el control del 14 de marzo los recursos necesarios para que el equilibrio militar en el país se incline hacia sus aliados. Por su parte, la reforma propuesta por Irán estaría focalizada en cambios menores en las distintas agencias de seguridad y en un reforzamiento limitado de las FAL, de tal modo que éstas sean capaces de responder a los desafíos de los pequeños grupos yihadistas en las regiones donde la presencia de Hezbollah es escasa o inexistente, pero sin que el mismo alcance unos niveles demasiado elevados que cuestionen la necesidad de que la milicia chií conserve sus armas como fuerza de disuasión y defensa del país. Curiosamente, la actitud estadounidense de impedir que las FAL se doten de los medios necesarios para convertirse en una fuerza de disuasión creíble en la frontera Sur no hace sino reforzar la posición política de Hezbollah como complemento imprescindible en dicha disuasión, un aspecto reconocido tanto por el Presidente Aoun como por numerosos altos mandos de las FAL.

Por otra parte, la actual tendencia de la Administración Trump (en compañía de Arabia Saudí) de colocar de nuevo a las FAL en una posición de enfrentamiento con Hezbollah no puede conducir más que a nuevas tensiones nacionales, además de complicar la labor de la FINUL, ignorando el importante papel desempeñado por ésta a la hora de calmar las tensiones en la frontera. Igualmente, los intentos de Hezbollah por dar a sus acciones una apariencia de colaboración con las FAL no hacen sino minar la credibilidad de éstas entre la comunidad suní. De hecho, cualquier avance en la convivencia en el Líbano no puede ignorar el carácter híbrido de su seguridad, consistente en la participación de agentes públicos y privados, libaneses y extranjeros, el cual se basa en unos equilibrios de poder actualmente existentes, que en caso de intentar de ser trastocados de manera unilateral no generarán más que un nuevo ciclo de violencia e inestabilidad en el país (Newby, 2016: 15).

\section{Conclusiones}

Un avance en la construcción de un sistema de seguridad que supere la rigidez del confesionalismo y que garantice su control democrático requerirá la creación de una confianza entre las partes que hoy por hoy no existe, además de un imprescindible consenso internacional que no parece probable en un momento de gran volatilidad regional. En ausencia de ello, los esfuerzos de la comunidad internacional deberían centrarse en mejorar la profesionalidad y capacidades del aparato policial con vistas a crear una policía judicial competente que refuerce el imperio de la ley y el respeto a los derechos humanos. Tales iniciativas no despertarían grandes rechazos por ninguna de las partes y tendrían la virtud de contribuir de forma genuina a la mejora de 
las condiciones de vida de los libaneses. Sin embargo, hasta ahora se ha hecho muy poco en este terreno, poniendo en evidencia que los países donantes de ayuda en el sector de la seguridad se han preocupado más de la salvaguarda de sus intereses geopolíticos que del progreso de la democratización en el Líbano, actuando bajo el paradigma de la securitización.

Por su parte, cualquier presión exterior para que las FAL se inclinen en uno u otro sentido en la arena política libanesa únicamente supone poner en peligro la unidad y credibilidad de la única institución de seguridad que posee un respaldo mayoritario en la sociedad. No obstante, una política de apoyo pragmático para dotarlas de los necesarios instrumentos que garanticen la defensa del país cumplirá la función de reforzar su credibilidad ante la población en una función ampliamente respaldada por el conjunto de las comunidades (Geha, 2015).

Mientras se construye el necesario consenso nacional para avanzar hacia un sistema de seguridad integrado bajo un control democrático, en las actuales circunstancias parece que el modelo de gobernanza híbrida característico del país seguirá estando vigente a medio plazo, por lo que será necesario que la comunidad internacional adapte su contribución a la reforma del sector de la seguridad a las necesidades de los libaneses, teniendo presente que cualquier alteración de los sutiles equilibrios de poder en el país puede conducir a su desestabilización.

\section{Bibliografía}

Azakir, M. (2016). "Saudi Arabia halts 3 billion dollars package to lebanese army, security aid", en Reuters, 19/02/2016. Disponible en: https://www.reuters.com/article/us-saudilebanon/saudi-arabia-halts-3-billion-package-to-lebanese-army-security-aid-idUSKCN0VS1KK

Barak, O. (2009). "Towards a representative military: The transformation of the lebanese officer corps since 1945", en The Middle East Journal, vol. 60, n 1, pp. 75-93.

Barak, O. (2009). The Lebanese Army: A National Institution in a Divided Society. Nueva York: SUNY Press.

Barnard, A. y M. Abi-Habib (2017). "Why Saad Hariri had that strange sojourn in Saudi Arabia", en The New York Times, 24/12/2017.

Belloncle, E. (2006). "Prospects of SSR in Lebanon", en Journal of Security Sector Management, vol. 4, pp. 3-19.

Boege, V. et al. (2009). "Hybrid political orders, not fragile states", en Peace Review, vol. 21, $\mathrm{n}^{\mathrm{o}} 1$, pp. 13-21.

Bryden, A. (2006). "Approaching privatization from a security governance perspective", en A. Bryden y M. Caparini (Eds.). Private actors and security governance. London: Transaction Publishers, pp. 3-22.

El Mufti, K. (2015). “Civil society's role in security sector reform in Lebanon”, en International Alert, Background Paper, pp. 1-12.

Fakhoury Mühlbacher, T. (2009). Democracy and power sharing in stormy weather: The Case of Lebanon. Wiesbaden: Verlag für Sozialwissenchaften.

Gaub, F. (2007). "Multi-Ethnic Armies in the Aftermath of Civil War: Lessons Learned from Lebanon", en Defence Studies, vol. 7, n 1, pp. 5-20.

Geha, C. (2015). “Citizen's perceptions of security institutions in Lebanon”, en International Alert, Background Paper, pp. 1-13. 
Gendzier, I. (2006). Notes from the minefield: United States Intervention in Lebanon and the Middle East, 1945-58. Nueva York: Columbia University Press.

Harris, A. (2007). "Bordering on the Impossible. Securing Lebanon's Borders with Syria”, en USI Journal, vol. 152, n 5, pp. 40-44.

International Crisis Group (2016). "Arsal in the Crosshairs. The Predicament of a Small Lebanese Town", en Middle East Briefing, no 46. Disponible en: https:/www.crisisgroup. org/middle-east-north-africa/eastern-mediterranean/lebanon/arsal-crosshairs-predicament-small-lebanese-border-town

Kechichian, J. (1985). "The Lebanese Army: Capabilities and challenges in the 1980s", en Conflict Quarterly, vol. 5, n 1, pp. 15-39.

Lefèvre, R. (2014). "The roots of crisis in Northern Lebanon", en Carnegie Middle East Center Disponible en: https://carnegieendowment.org/files/crisis_northern_lebanon.pdf

Lion Bustillo, J. (2014). “Líbano y Siria: ¿Entre la disociación y el desbordamiento?”, en Revista CIDOB d'Afers Internacionals, n 108, pp. 223.

Lion Bustillo, J. (2017). "El Estado Islámico y Jabhat al-Nusra, ¿Nuevos actores en el Líbano?", en Revista del Instituto Español de Estudios Estratégicos, n. 9, pp. 105-132. Disponible en: http://revista.ieee.es/index.php/ieee/article/view/281/483

Malka, A. (2008). "Israel and Asymmetrical Deterrence", en Comparative Strategy, vol. 27, $\mathrm{n}^{\mathrm{o}} 1$, pp. 1-19.

McLaurin, R. (1991). "From professional to political: The redecline of the Lebanese army", en Armed Forces and Society, vol. 17, n 4, pp. 545-568.

Nashabe, O. (2009). The Arab reform initiative security sector reform in Lebanon internal security forces and general security. Retrieved from the Arab Reform Initiative website: https://gsdrc.org/document-library/security-sector-reform-in-lebanon-internal-securityforces-and-general-security/

Nerguizian, A. (2009). The Lebanese armed forces: Challenges and opportunities in PostSyria Lebanon. Washington: Center for Strategic and International Studies.

Nerguizian, A. (2017). The Lebanese armed forces, Hezbollah and military legitimacy. Washington: Center for Strategic and International Studies.

Newby, V. (2016). "Positive hybridity? Unpacking UN security sector reform in South Lebanon", en Contemporary Politics, vol. 23, n 2, pp. 1-19.

Picard, E. (1999). The demobilisation of Lebanese militias. Oxford: Center for Lebanese Studies.

Richmond, O. P. (2015). "The dilemmas of a hybrid peace: Negative or positive?", en Cooperation and Conflict, vol. 50, $\mathrm{n}^{\circ}$ 1, pp. 50-68.

Rougier, B. (2015). The Sunni Tragedy in the Middle East: Northern Lebanon from Al Qaeda to ISIS. Princeton: Princeton University Press.

Salem, P. (2012). "Can Lebanon survive the Syrian crisis?" The Carnegie Papers. Disponible en: https://carnegieendowment.org/files/lebanon_syrian_crisis.pdf

Saliba, A. (2012). "Security sector in Lebanon: Jurisdiction and organization", Carnegie Endowment for Peace, 2012. Disponible en: http://carnegieendowment.org/files/Security Sector_in_Lebanon2.pdf

Schroeder, U. et al. (2014). "Security sector reform and the emergence of hybrid security governance", en International Peacekeeping, vol. 21, n 2, pp. 214-30.

Siegman, H. (2000). "Being Hafiz al-Assad: Syria's chilly but consistent peace strategy", en Foreign Affairs, vol. 79, n 3, pp. 2-7.

Sigaud, D. (1988). "L'armée libanaise: Éclatement ou destin national?", en Cahiers de l'Orient, $\mathrm{n}^{\mathrm{o}} 11$, pp. 47-68. 
Taureck, R. (2006). "Securitization Theory and securitization studies", en Journal of International Relations and Development, vol. 9, $\mathrm{n}^{\circ}$ 1, pp. 53-61.

Waever, O. (1995). “Securitization and Desecuritization”, en R. D. Lipchutz. (ed.). On Security. Nueva York: Columbia University Press, pp. 46-86.

Sayigh, Y. (2009). "Fixing broken windows: Security sector reform in Palestine, Lebanon, and Yemen", en Research Paper, $\mathrm{n}^{\circ}$ 17. Disponible en: http://carnegieendowment.org/files/security_sector_reform.pdf

Tholens, S. (2017). "Border management in an era of 'statebuilding lite': Security assistance in Lebanons hybrid sovereignty", en International Affairs, vol. 93, n 4, pp. 865-882.

Van Veen, E. (2015). "Elites, power and security: How the organization of security in Lebanon serves elite interests", Clingendael CRU Report.

Wulf, H. (2007). Challenging the weberian concept of the state: The future of the monopoly of violence. ACPACS Occasional Papers Online, $\mathrm{n}^{\circ}$ 9. Brisbade: The Australian Centre Peace and Conflict Studies.

Wulf, H. (2011). "Security sector reform in developing and transitional countries revisited", en B. Austin et al., Advancing conflict transformation: The berghof handbook II. Opladen/Framington Hills: Barbara Budrich Publishers, pp. 338-357.

Ziadeh, R. (2011). Power and policy in Syria: Intelligence services, foreign relations and democracy in the modern Middle East. London: I.B. Tauris. 